\title{
Audit of documentation proficiency of emergency department patients who are discharged against medical advice before and after implementation of a checklist
}

Sze Joo Juan*, Ghee Hian Lim, Beng Leong Lim

Emergency Department, Ng Teng Fong General Hospital, Singapore

Received: April 6, 2016

DOI: $10.5430 /$ jha.v $5 \mathrm{n} 4 \mathrm{p} 28$
Accepted: April 21, 2016

URL: http://dx.doi.org/10.5430/jha.v5n4p28

\begin{abstract}
Objective: Documentation of the discharge against medical advice (AMA) is poorly performed in the emergency department (ED). Little is known about the impacts of a checklist on this. Our study aimed to compare the quality of AMA documentation before and after implementation of a checklist.

Methods: A retrospective review was conducted followed by a prospective study; each over three months of AMA interactions in our ED pre and post implementation of a checklist. An 11-point checklist was used to determine documentation quality during these two periods. Quality was assessed based on the number of points fulfilled on this tool. Documentation was classified as "good" (8-11), "average" (4-7) and "poor" (0-3). The primary outcome measured was the proportions of discharged AMA records that showed "good", "average" and "poor" documentation. Secondary outcomes were compliance rates to each of the categories of the checklist before and after its use.

Results: 339 and 309 complete records were retrieved from the retrospective and prospective arms respectively. The proportions of case records in the three grades before and after use of the checklist respectively were: poor, 199/339 (59\%) vs. 7/313 (2\%); fair, $133 / 339(39 \%)$ vs. $66 / 313(21 \%)$ and good $7 / 339(2 \%)$ vs. $240 / 313$ (77\%); all $p$-values were statistically significant. There were also statistically significant differences in compliance rates to each of the categories of the checklist pre and post checklist implementation.

Conclusions: This study shows improvement in quality and compliance rates in the audit categories after the implementation of an AMA checklist.
\end{abstract}

Key Words: Against medical advice, Checklist, Compliance

\section{INTRODUCTION}

Discharge against medical advice (AMA) is not an uncommon occurrence in the Emergency Department (ED). Approximately $1 \%-2 \%$ of discharges at ED in the United States are
AMA; this figure goes up to $6 \%$ in disadvantaged inner-city populations. $^{[1]}$

Southern, Nahvi \& Arnsten ${ }^{[2]}$ have demonstrated that inpatients whom leave AMA have higher 30-day re-admission

\footnotetext{
*Correspondence: Sze Joo Juan; Email: Sze_Joo_Juan@juronghealth.com.sg; Address: Emergency Department, Ng Teng Fong General Hospital, 1 Jurong East Street 21, 609606, Singapore.
} 
rates (odds ratio $=1.84,95 \%$ CI: $1.69-2.01$ ) and increased risk of mortality (odds ratio $=2.05,95 \%$ CI: $1.48-2.86$ ).

Emergency physicians (EPs) must make every attempt to prevent patients from leaving AMA. In situations when AMA discharge is unavoidable, the key is to optimize legal protection for the staff executing the AMA. ${ }^{[1]}$

The complex ED environment poses significant challenges. Time constraints, unpredicted interruptions, diagnostic uncertainty, staff shift changes and crowding frequently undermine good intentions. ${ }^{[3]}$

It is important that this informed communication immediately preceding discharge AMA is clearly documented despite these challenging ED constraints. ${ }^{[1]}$

This communication and documentation process must balance reliability and efficiency gains provided by standardization with the flexibility required to be effective across a wide range of patients' literacy levels and cultural backgrounds in a cosmopolitan country like Singapore.

Schaefer \& Monico ${ }^{[4]}$ showed, in their retrospective single center cohort study of healthcare provider documentation of adult patients who left an ED AMA over one year, that there is suboptimal documentation in AMA cases by clinicians.

Properly executed AMA forms and procedures do not completely insulate EPs from liability in a medical malpractice action. It can however provide important legal protection. The AMA records evidence of refusal of care and its documentation can provide vital evidence for any ensuing litigation. ${ }^{[1]}$

The use of an AMA discharge checklist provides standardization of practice and a visual reminder to doctors of the critical steps in their communication with the patient.

The aim of our study is to compare the documentation proficiency of discharge AMA before and after implementation of an AMA discharge checklist.

\section{Methods}

We conducted a retrospective review of AMA interactions within our ED from $1^{\text {st }}$ March 2013 to $31^{\text {st }}$ May 2013 and a prospective study of AMA interactions post implementation of an AMA checklist from $1^{\text {st }}$ December 2013 to $28^{\text {th }}$ February 2014.

Prior to the implementation of the AMA checklist in the retrospective arm, documentation of AMA was based on the discretion of the individual physician. Our ED implemented an 11 point AMA checklist during the prospective arm to guide the attending physicians. Two independent reviewers audited the retrospective arm using this checklist.
The study hospital is a 400 bed acute adult general hospital with 24-hour EPs' cover. It is a tertiary hospital that receives referrals from all over the country. Our institutional ethics committee had approved this study for waiver of consent.

\subsection{Sampling}

In the retrospective arm, we searched our departmental electronic database and included all cases that have a designated final disposition status of "discharge AMA". Two independent EPs reviewed the electronic records and they supplemented data collection using our hospital electronic databases.

In the prospective arm, the ED doctors were asked to fill in the AMA checklist for patients who were discharged AMA.

In this arm, we recruited a consecutive sample. Exclusion cases included those with missing and incomplete checklists.

Data collected in both arms included the demographics of the patients and the Patient Acuity Category (PAC) status of the cases. The criteria for the various PAC categories are shown in Table 1 recommended by the Society of Emergency Medicine of Singapore. We have included the various PAC status of the cases to see if there was marked difference in the distribution of the acuity of patients who discharge AMA in the pre and post intervention group.

\subsection{Intervention using AMA checklist}

We performed a literature search for the essentials of documentation of AMA. The hospital existing policy for AMA was also studied and discussed with the departmental senior EPs. The 11-point AMA checklist was adopted from the literature and after a consensus among these senior ED clinicians. The AMA checklist was circulated amongst the senior EP clinicians and feedback was gathered before the checklist was finalized and used. Table 2 shows the components of this AMA checklist.

Following the data analysis of the retrospective arm, the existing standards of discharge AMA documentation were presented to the ED medical and nursing staff during departmental meetings and teachings.

The importance and components of a good AMA interaction and documentation, together with the 11-point AMA checklist, were shared with them during these sessions and via electronic mails. The checklist served as a visual reminder of all the salient points that needed to be discussed with the patient/family and thereafter documented.

Prior to the prospective arm, the ED staff were informed that they had to complete the AMA checklist for all patients who decided to discharge AMA. The nursing staff would ensure 
that this form was completed before he/she discharged the patient. This checklist was then filed with the printed copy of the patient's ED electronic records.
There was a wash out period of one month following ED staff briefing and education using the checklist till commencement of the prospective arm.

Table 1. PAC system as recommended by Society of Emergency Medicine of Singapore

PAC Criteria

1. In or with imminent cardiorespiratory arrest requiring immediate attention

2. Not in imminent cardiorespiratory arrest but require early attention (within $80 \mathrm{~min}$ ); failing which deterioration is likely

3. Ambulatory patients with mild to moderate symptoms

4. Non-emergency patients who can be managed in the primary care setting

Table 2. The 11-point AMA checklist

$$
\begin{aligned}
& \text { - Mental capacity } \\
& \text { - Diagnosis explained } \\
& \text { - Treatment explained } \\
& \text { - Implication of no treatment } \\
& \text { - What to look out for } \\
& \text { - When to return } \\
& \text { - Family involvement } \\
& \text { - Alternative treatment } \\
& \text { - Follow up care } \\
& \text { - Document that patient understands }
\end{aligned}
$$

Note. Quality of documentation: Poor (0-3), Average (4-7), Good (8-11)

\subsection{Outcome measures}

We used the 11-point checklist to determine the quality of the AMA documentation.

A consensus was reached among the senior EPs that the quality of AMA documentation would be classified based on number of points fulfilled on the 11-point tool. The documentation could be classified as "good" (8-11), "average" (4-7) and "poor" (0-3).

The primary outcomes measured were the proportions of discharged AMA records that showed "good", "average" and "poor" documentation before and after implementation of the AMA checklist. Secondary outcomes were compliance rates to each of the 11 audit categories before and after implementation of the checklist.

\subsection{Data analysis}

Data was entered into a Microsoft Excel spreadsheet and then transposed into SPSS version 21 for analysis.
Data was analyzed using descriptive statistics. We reported medians with their interquartile ranges (IQRs). Chi-square and Fisher's exact tests were used to compare the outcomes in the retrospective and prospective arms. $P$-values were two tailed and $p<.05$ were considered statistically significant.

\section{RESULTS}

\subsection{Recruitment}

We retrieved 339 case records from $1^{\text {st }}$ March 2013 to $31^{\text {st }}$ May 2013 and 317 records from $1^{\text {st }}$ December 2013 to $28^{\text {th }}$ February 2014. This represented $2.5 \%$ and $2.3 \%$ of all ED attendances respectively. There was no missing data in the pre intervention group. There were four incomplete records (1.3\%) in the post intervention group and 313 of them were included in the final analysis. Patient demographics, diagnosis code, distribution of acuity of cases and seniority of doctors were similar in the pre and post intervention groups. Table 3 shows the baseline features of the patients during these two periods.

\subsection{Quality of records and compliance rates to audit cat- egories}

Table 4 reveals the number of AMA cases with "poor", "average" and "good" scores before and after the checklist implementation. The percentage of case records that demonstrated good documentation rose from $2 \%$ to $77 \%$ after the intervention $(p<.001)$.

Table 5 shows the compliance rates to each of the 11 audit categories before and after implementation of the checklist. There was improvement in the compliance rates in all the audit categories. Documentation of the mental capacity of the patients showed the most marked improvement. In the pre intervention group, $2.1 \%$ documented the assessment of mental capacity. This increased to $94.9 \%$. in the post intervention group. The three categories that were most important to clinical practice also showed significant improvement. The 
percentage that informed the patient of the implications of list. Similarly, documenting that the patients understand the no treatment, what to look out for upon discharge and when entire AMA process is important and there was a marked to return, improved from less than $50 \%$ to $80.8 \%, 71.6 \%$, improvement from $15.3 \%$ to $97.1 \%$ post intervention. These $73.8 \%$ respectively after the introduction of the AMA check- results were statistically significant.

Table 3. Demographics, PAC status, diagnosis code and seniority of doctors for pre and post intervention groups

\begin{tabular}{|c|c|c|}
\hline & Pre intervention [N = 339, n (\%)] & Post intervention $[\mathrm{N}=313, \mathrm{n}(\%)]$ \\
\hline \multicolumn{3}{|l|}{ Age } \\
\hline Median (IQR) & $44.0(25)$ & $43.0(29)$ \\
\hline \multicolumn{3}{|l|}{ Gender } \\
\hline - Male & $228(67.3)$ & $198(63.3)$ \\
\hline - Female & $111(32.7)$ & $115(36.7)$ \\
\hline \multicolumn{3}{|l|}{ PAC } \\
\hline - PAC 1 & $4(1.2)$ & $6(1.9)$ \\
\hline - PAC 2 & $226(66.6)$ & $227(72.5)$ \\
\hline - PAC 3 & $109(32.2)$ & $80(25.6)$ \\
\hline \multicolumn{3}{|l|}{ Diagnosis code } \\
\hline - Medical & $224(71.6)$ & $218(64.3)$ \\
\hline - Surgical & $87(27.8)$ & $118(34.8)$ \\
\hline - Undifferentiated & $2(0.6)$ & $3(0.9)$ \\
\hline \multicolumn{3}{|l|}{ Ethnic groups } \\
\hline - Chinese & $150(44.2)$ & $145(46.3)$ \\
\hline - Malay & $63(18.6)$ & $70(22.4)$ \\
\hline - Indian & $102(30.1)$ & $88(28.1)$ \\
\hline - Others & $24(7.1)$ & $10(3.2)$ \\
\hline \multicolumn{3}{|c|}{$\begin{array}{l}\text { Concordance of ethnic group between } \\
\text { patient and doctor }\end{array}$} \\
\hline - Yes & $124(36.6)$ & $109(34.8)$ \\
\hline - No & $215(63.4)$ & $204(65.2)$ \\
\hline \multicolumn{3}{|l|}{ Seniority of doctors } \\
\hline - Senior & $15(4.4)$ & $8(2.6)$ \\
\hline - Junior & 324 (95.6) & 305 (97.4) \\
\hline
\end{tabular}

Note. IQR: Interquartile ranges; PAC: Patient Acuity Category; Diagnosis code: Medical cases include diseases of the respiratory, cardiovascular, digestive, genitourinary, skin and nervous systems; Surgical cases include general surgery, orthopedics, neurosurgical conditions; Seniority of doctors: Senior refers to Emergency Medicine specialist; Junior refers to non Emergency Medicine specialist

Table 4. Comparison of quality of documentation pre and post intervention

\begin{tabular}{llll}
\hline & Pre intervention [N = 339, $\mathbf{n}(\%)]$ & Post intervention [N = 313, $\mathbf{n}(\%)]$ & $p$-value \\
\hline Poor & $199(59)$ & $7(2)$ & $<.001$ \\
Fair & $133(39)$ & $66(21)$ & $<.001$ \\
Good & $7(2)$ & $240(77)$ & $<.001$ \\
\hline
\end{tabular}

Note. Quality of documentation: Poor (0-3); Average (4-7); Good (8-11)

\section{Discussion}

AMA discharge continues to be a prevalent and frustrating problem for patients and their physicians. The literature is limited primarily to medical record reviews and retrospective analyses of associations with AMA discharge. Data for physicians on how to effectively manage and intervene in these complicated patient encounters is scant. ${ }^{[5]}$

Our study shows that there was improvement in the qual- ity and compliance rates in all the audit categories of AMA documentation after the implementation of the checklist.

Our results in the pre-intervention group are similar to a study done by Schaefer \& Monico, 2013. ${ }^{[4]}$ It demonstrates that the ED clinicians are not sufficiently documenting the appropriate quality or quantity of information needed to make fully informed decisions. They also reported that the reason for this insufficient documentation may be that clinicians lack knowledge of what to document. 
Table 5. Compliance rates to components of the 11-point audit

\begin{tabular}{|c|c|c|c|}
\hline & Pre intervention $[\mathrm{N}=339, \mathrm{n}(\%)]$ & Post intervention $[\mathrm{N}=313, \mathrm{n}(\%)]$ & $p$-value \\
\hline Mental capacity & $7(2.1)$ & $297(94.9)$ & $<.001$ \\
\hline Diagnosis explained & 203 (59.9) & $291(93.0)$ & $<.001$ \\
\hline Reasons for AMA & $165(48.7)$ & $273(87.2)$ & $<.001$ \\
\hline Treatment explained & $182(53.7)$ & $290(92.7)$ & $<.001$ \\
\hline Implication of no treatment & $63(49.3)$ & $253(80.8)$ & $<.001$ \\
\hline What to look out for & $73(21.5)$ & $224(71.6)$ & $<.001$ \\
\hline When to return & $80(23.6)$ & $231(73.8)$ & $<.001$ \\
\hline Family involvement & $93(27.4)$ & $145(46.3)$ & $<.001$ \\
\hline Alternative treatment & $39(11.5)$ & $231(73.8)$ & $<.001$ \\
\hline Follow up care & $210(61.9)$ & $236(75.4)$ & $<.001$ \\
\hline Documents that patient understands & $52(15.3)$ & $304(97.1)$ & $<.001$ \\
\hline
\end{tabular}

There is limited literature concerning the usefulness of an AMA checklist. We postulate that the improvement seen in the post-intervention group is likely that the discharge AMA checklist provides standardization of practice and a visual reminder to the doctors of the critical steps in their communication with the patient, and then documenting the process. Our postulation concurs with what is reported in the literature. ${ }^{[6]}$

Assessing the mental capacity of the patient shows the most marked improvement in the post intervention group. When patients sign out AMA, they must first be deemed to have decision-making capacity. They need to be able to express their choices and demonstrate an understanding of relevant information. ${ }^{[1]}$ There are some potential reasons for the relative lack of documentation of this critical aspect. Providers may not realize that they should be documenting mental capacity, especially since it is often quite apparent that the patient has full capacity to make decisions. In addition, the providers may not know how to test for adequacy of mental capacity. ${ }^{[4]}$

The three categories that are important to clinical practice also showed significant improvement in the post intervention group i.e. informing the patient of the implications of no treatment, what to look out for upon discharge and when to return to ED.

Studies ${ }^{[7,8]}$ reported that patients often leave the ED with an incomplete understanding of their care and instructions. This increases their risk for adverse events, unscheduled ED visits and hospitalization. Following discharge AMA, patients need to be aware of the red flags that warrant their return to the ED. This will help minimize adverse outcomes. ${ }^{[9]}$

AMA discharge is associated with increased risks of mortality and 30-day re-admission. ${ }^{[2]}$ It is hence especially im- portant for patients who discharge AMA to be informed and understand the first warning symptoms of clinical deterioration following discharge that should prompt a return visit to the ED for reassessment and treatment.

There is currently no study to show whether an AMA checklist can reduce mortality and 30 day re-attendance rates. We are looking into studying the clinical impact that our checklist have on re-attendances and mortality in the ED.

We have presented our study findings during a clinical meeting to the chairman of our medical board. The AMA checklist is now incorporated into the electronic patient record system and will be used for all patients who are discharged AMA throughout our institution.

\section{Limitations}

There is a retrospective arm in our study. The available data may be subjected to interpretation bias. We have tried to minimize this by using two investigators to review the records.

It is also difficult to determine causation and only association can be established. This study was based on a single hospital and our results may not be generalizable to other EDs.

The auditors were not blinded to the use of the checklist and the study's goal. This could introduce bias in assessing compliance to the 11-point AMA checklist in the pre-intervention group.

We have made the extrapolation that improved documentation translated to improvement of actual discussion of the AMA process between the clinicians and the patients. It will be worthy of our future research to study if this process indeed did improve patients' understanding of the AMA process. We are monitoring the actual compliance rates to this AMA checklist outside study condition. 
Although it is possible other education processes occurring outside our study might have impacted on the improvement of AMA documentation after checklist implementation, we believed that this is very unlikely. Also, we did not explore whether an AMA checklist will affect ED re-attendances or adverse events in our study. This is also an important clinical endpoint that is worthy of our future research.

\section{Conflicts of InTEREST Disclosure}

The authors declare they have no conflict of interest.

\section{REFERENCES}

[1] Levy F, Mareiniss DP, Iacovelli C. The Importance of a Proper Against-Medical-Advice (AMA) Discharge: How Signing Out AMA May Create Significant Liability Protection for Providers. Journal of Emergency Medicine. 2012; 43(3): 516-520. PMid: 21715123. http://dx.doi.org/10.1016/j.jemermed.2011.05.030

[2] Southern WN, Nahvi S, Arnsten JH. Increased risk of mortality and readmission among patients discharged against medical advice. American Journal of Medicine. 2012; 125(6): 594-602. PMid: 22513194. http://dx.doi.org/10.1016/j.amjmed.2011.12.017

[3] Samuels-Kalow ME, Stack AM, Porter SC. Effective Discharge Communication in the Emergency Department. Annals of Emergency Medicine. 2012; 60(2): 152-159. PMid: 22221840. http: //dx.doi.org/10.1016/j.annemergmed.2011.10.023

[4] Schaefer MR, Monico EP. Documentation proficiency of patients who leave the emergency department against medical advice. Connecticut Medicine. 2013; 77(8): 461-6. PMid: 24156173.

[5] David JA. "I'm Going Home": Discharges Against Medical Advice. Mayo Clin Proc. 2009.
[6] Hales B, Terblanche M, Fowler R, et al. Development of medical checklists for improved quality of patient care. International Journal for Quality in Health Care Journal of the International Society for Quality in Health Care. 2008; 20(1): 22-30(9).

[7] Engel KG, Buckley BA, Mccarthy DM, et al. Communication amidst chaos: Challenges to patient communication in the emergency department. Journal of Clinical Outcomes Management Jcom. 2010; 17(10): 17-21.

[8] Engel KG, Heisler M, Smith DM, et al. Patient comprehension of emergency department care and instructions: are patients aware of when they do not understand? Annals of Emergency Medicine. 2009; 53(4): 454-461. e15. PMid: 18619710. http://dx.doi.org/10. 1016/j . annemergmed. 2008.05.016

[9] Kristen GE, Barbara AB, Victoria EF, et al. Patient Understanding of Emergency Department Discharge Instructions: Where are Knowledge Deficits Greatest? Society for Academic Emergency Medicine. 2012. 